BMJ Open

Diabetes

Research

\& Care

\title{
Physical activity and cardiometabolic health in adolescents with type 2 diabetes: a cross-sectional study
}

\author{
Jana L Slaght, ${ }^{1,2}$ Brandy Alexandra Wicklow (1) , 1,2,3 Allison B Dart,, ${ }^{1,2}$ \\ Elizabeth A C Sellers, ${ }^{1,2,3}$ Melissa Gabbs, ${ }^{1,2}$ Marylin Carino, , ${ }^{1,2}$ \\ Jonathan M McGavock (i) 1,2
}

To cite: Slaght JL, Wicklow BA, Dart AB, et al. Physical activity and cardiometabolic health in adolescents with type 2 diabetes: a cross-sectional study. BMJ Open Diab Res Care 2021;9:e002134. doi:10.1136/ bmjdrc-2021-002134

- Supplemental material is published online only. To view, please visit the journal online (http://dx.doi.org/10.1136/ bmjdrc-2021-002134).

Received 12 January 2021 Revised 14 April 2021 Accepted 25 April 2021

\section{Check for updates}

(c) Author(s) (or their employer(s)) 2021. Re-use permitted under CC BY-NC. No commercial re-use. See rights and permissions. Published by BMJ.

${ }^{1}$ Pediatrics and Child Health, University of Manitoba Children's Hospital Research Institute of Manitoba, Winnipeg, Manitoba, Canada

${ }^{2}$ Diabetes Research Envisioned and Accomplished in Manitoba (DREAM) Theme, Children's Hospital Research Institute of Manitoba, Winnipeg, Manitoba, Canada

${ }^{3}$ Diabetes Research Envisioned and Accomplished in Manitoba (DREAM) Theme, Pediatric Endocrinology and Metabolism, Department of Paediatrics and Child Health, University of Manitoba, Winnipeg, Manitoba, Canada

Correspondence to Dr Jonathan M McGavock; jmcgavock@chrim.ca

\section{ABSTRACT}

Introduction Youth living with type 2 diabetes display increased risk of cardiovascular disease (CVD). It is unclear if regular physical activity (PA) modifies this risk.

Research design and methods We compared CVD risk factors in a cross-sectional study of 164 youth with type 2 diabetes stratified according to weekly vigorous-intensity PA. Outcomes were hemoglobin A1c ( $\mathrm{HbA1C})$, ambulatory blood pressure (BP; ambulatory 24-hour readings), plasma lipoproteins, and albuminuria. The main exposure, vigorous-intensity PA, was quantified with the Adolescent Physical Activity Recall Questionnaire.

Results Youth were $15 \pm 3$ years, and $78 \%$ lived rurally and $68 \%$ were female, with a mean body mass index (BMI) Z-score of $2.4 \pm 1.1$ and a mean $\mathrm{HbA1c}$ of $9.6 \% \pm 2.6 \%$. Youth who participated in regular vigorous-intensity PA (40\%; $n=67)$ achieved nearly twice the dose of PA than peers who did not (62 vs 34 metabolic equivalent score-hour/week, $p=0.001$ ). After adjusting for duration of diabetes, BMI Z-score, sex, and smoking, youth who engaged in vigorous-intensity PA displayed lower $\mathrm{HbA} 1 \mathrm{c}$ (9.1\% vs $9.9 \%, p=0.052)$, diastolic BP $(70 \mathrm{~mm} \mathrm{Hg}$ vs 73 $\mathrm{mm} \mathrm{Hg}, \mathrm{p}=0.002$ ), diastolic load ( $20 \%$ vs $26 \%, p=0.023$ ), and mean arterial pressure $(87.3 \mathrm{~mm} \mathrm{Hg}$ vs $90.3 \mathrm{~mm} \mathrm{Hg}$, $\mathrm{p}<0.01)$, compared with youth who did not. Compared with youth who did not participate in regular vigorous-intensity PA, those who did also displayed lower odds of albuminuria after adjusting for duration of diabetes, sex, smoking, rural residence, and BMI Z-score (adjusted OR: $0.40,95 \% \mathrm{Cl}$ 0.19 to 0.84 ).

Conclusions Among youth with type 2 diabetes, participation in vigorous-intensity PA is associated with lower CVD risk.

\section{INTRODUCTION}

Management of cardiometabolic risk among adolescents with type 2 diabetes is a significant clinical challenge. Therapeutic trials of behavioral lifestyle modification are limited, ${ }^{1-3}$ but suggest that health behavior change is not efficacious for long-term cardiometabolic risk management in many adolescents with type 2 diabetes. Within these trials, however, some adolescents achieve and sustain favorable cardiometabolic risk profiles, while others do not. ${ }^{45}$ The factors that contribute

\section{Significance of this study}

What is already known about this subject?

- Regular physical activity, particularly vigorousintensity physical activity, is associated with lower cardiometabolic risk profile in adolescents.

- It remains unclear if regular physical activity, particularly vigorous-intensity physical activity, is associated with favorable cardiometabolic health profiles among adolescents living with type 2 diabetes.

What are the new findings?

- Greater participation in vigorous-intensity physical activity is associated with better cardiometabolic health, particularly lower ambulatory blood pressure and reduced odds of albuminuria, in adolescents living with type 2 diabetes.

How might these results change the focus of research or clinical practice?

- These results support clinical practice guidelines for promoting regular physical activity, particularly vigorous intensity, to manage cardiometabolic risk factors in adolescents living with type 2 diabetes.

to a favorable cardiometabolic health profile among adolescents with type 2 diabetes remain poorly understood.

Physical activity is a cornerstone in the management of type 2 diabetes. ${ }^{6}$ Current clinical practice guidelines recommend regular daily physical activity for adolescents living with type 2 diabetes. ${ }^{7-9}$ Despite these recommendations, our group ${ }^{10}$ and others ${ }^{11} 12$ demonstrated that adolescents with type 2 diabetes achieve less daily physical activity than their peers without type 2 diabetes. Adolescents without diabetes who engage in regular daily physical activity, ${ }^{13}$ particularly vigorous-intensity physical activity, ${ }^{14}{ }^{15}$ display better cardiometabolic profiles compared with adolescents who do not. Two previous cross-sectional studies revealed that adolescents who engage in 
physical activity more than 2 days per week display better glycemic control and some cardiometabolic parameters. ${ }^{16}{ }^{17}$ However these studies did not account for the intensity of physical activity, include ambulatory blood pressure measurements nor did they assess renal function. Therefore, it remains unclear if regular physical activity, particularly vigorous-intensity physical activity, is associated with better cardiometabolic risk profiles among adolescents living with type 2 diabetes.

The current study was designed to determine the association between physical activity and cardiometabolic health outcomes in adolescents with type 2 diabetes. Using a cross-sectional design embedded within a large cohort study, we tested the hypothesis that adolescents who participate in regular vigorous-intensity physical activity will display a better cardiometabolic health profile, compared with those who do not participate in regular vigorous-intensity physical activity.

\section{DESIGN AND METHODS}

\section{Study design, setting, and participants}

To test the study hypothesis, we stratified adolescents with type 2 diabetes who are participating in the improving renal Complications in Adolescents with type 2 diabetes through REsearch (iCARE) cohort study ${ }^{18}$ according to their participation in regular vigorous-intensity physical activity (Clinical Trials Registry: NCT02818192). We followed the Strengthening the Reporting of Observational Studies in Epidemiology guidelines to report the results of these analyses. ${ }^{19}$ Adolescents aged $10-18$ years with a diagnosis of type 2 diabetes (according to Diabetes Canada Clinical Practice Guidelines ${ }^{8}$ and absence of diabetes-related autoantibodies) ${ }^{20}$ were recruited into the iCARE cohort study during a routine clinical visit to the pediatric diabetes clinic at Winnipeg Children's Hospital. The iCARE cohort is a prospective observational study of the biopsychosocial factors associated with albuminuria in youth with type 2 diabetes diagnosed before 18 years of age. ${ }^{18}$ Recruitment for this substudy began in November 2011 and ended in July 2016. Recruitment for the larger cohort is ongoing, with a current sample size of 355 youth with type 2 diabetes. Exclusion criteria for the cohort study are (1) diabetes secondary to medication use or surgery, (2) cancer, (3) evidence of alcohol or drug abuse, (4) patient or caregiver unable or unwilling to provide voluntary informed assent/consent, and (5) current pregnancy. For this particular analysis, participants who did not complete the required physical activity questionnaire due to a protocol change in 2016 were not included. Every parent or guardian gave written informed consent before enrollment, and every youth gave written informed consent in accordance with the Declaration of Helsinki (HREB \#HS13255). The study design is also guided by the iCARE participant and family advisory group in accordance with the current guidelines for participatory action research with Indigenous populations. ${ }^{21}$

\section{Exposure of interest}

The main exposure of interest was regular participation in vigorous-intensity physical activity. Physical activity was determined with a validated 12-month physical activity recall instrument (Adolescent Physical Activity Recall Questionnaire) ${ }^{22}$ This instrument categorizes physical activity into questions regarding organized and non-organized activities during the winter and summer months. Adolescents report activities, which are assigned metabolic equivalent (MET) score using the Compendium of Physical Activities. ${ }^{23}$ The metabolic equivalent for each activity was then multiplied by the weekly frequency and duration of participation to determine total MET-hour/week. Activities were stratified into light (<3 METs), moderate (3.1-5.9 METs), or vigorous ( $>6$ METs) intensity in accordance with previous guidelines. ${ }^{24}$ Organized and non-organized activities were considered separately. Regular participation was defined as self-reporting participation $\geq 3$ times per week. Examples of light-intensity and vigorous-intensity organized and nonorganized activities including the estimated MET values are provided in table 1.

\section{Outcomes of interest}

The main outcome measures were hemoglobin Alc (HbAlc), 24-hour ambulatory blood pressure, albuminuria (first morning sample), and serum cholesterol and lipoproteins. HbAlc and serum cholesterol and lipoproteins were drawn after a minimum 10-hour fast and measurements performed by the Shared Health Manitoba Diagnostic Laboratories using the Roche Cobas Integra 800 CTS and Cobas c513. An HbA1c $>9.0 \%$ was treated as a binary outcome

Table 1 Examples of activities reported by adolescents with type 2 diabetes and the associated MET

\begin{tabular}{|c|c|c|c|c|c|}
\hline Organized activity & MET & $\begin{array}{l}\text { Number } \\
\text { reporting }\end{array}$ & $\begin{array}{l}\text { Average MET min/ } \\
\text { week/person }\end{array}$ & $\begin{array}{l}\text { Average } \mathrm{min} / \text { week/ } \\
\text { person }\end{array}$ & $\%$ participation \\
\hline Gym class & 6.0 & 73 & 1294 & 216 & 45 \\
\hline Volleyball & 5.2 & 41 & 1327 & 255 & 25 \\
\hline Ice hockey & 8.0 & 43 & 637 & 80 & 26 \\
\hline Soccer & 8.4 & 27 & 1391 & 166 & 16 \\
\hline Basketball & 7.3 & 50 & 1323 & 181 & 30 \\
\hline
\end{tabular}

MET is metabolic equivalent, which is a measure of the metabolic cost associated with the activity: $1 \mathrm{MET}=3.5 \mathrm{~mL} / \mathrm{kg} / \mathrm{min}$.

Number reporting is the number of participants in the cohort that recalled participating in that form of organized physical activity. 
of poor glycemic control. Participants completed 24-hour ambulatory blood pressure monitoring using SpaceLabs monitors (Snoqualmie, Washington, USA). Values were standardized to sex and height. ${ }^{25}$ Daytime, night-time, and 24-hour values for systolic, diastolic and mean arterial pressures were treated as continuous variables. Mean blood pressure values $\geq 95$ th centile combined with daytime and nocturnal systolic and diastolic blood pressure loads $>25 \%$ were used to classify hypertension (ie, the per cent of time spent above the 95th centile for sex and height).$^{25}$ Overnight or first morning urine was collected for evaluation of albuminuria. Albumin to creatinine ratio was treated as a continuous variable, and albuminuria was defined as urine albumin to creatinine ratio greater than or equal to $2.0 \mathrm{mg}$ / mmol. ${ }^{26}$

\section{Confounding and demographic variables}

Anthropometric measurements were performed in duplicate without shoes and in light indoor clothing and averaged. Body mass (weight) was measured to the nearest 0.1 $\mathrm{kg}$ on a calibrated digital scale and height was measured to the nearest $0.1 \mathrm{~cm}$ with a stadiometer (Healthometer, Illinois, USA). Body mass index (BMI) was calculated as mass (in $\mathrm{kg}$ ) divided by height squared $\left(\mathrm{m}^{2}\right)$. Age-specific and sex-specific Z-scores were calculated according to the WHO growth charts. ${ }^{27}$ Overweight status was defined as BMI between the 85th and 97th centile for age and gender, which is equivalent to a BMI Z-score of 1 to $<2$. Obese status was defined as BMI $\geq 97$ th centile for age and gender or a BMI Z-score of $\geq 2 .{ }^{28}$ For participants over 18 years of age during the study visit, BMI-based criteria of $25-29.9 \mathrm{~kg} / \mathrm{m}^{2}$ and $\geq 30 \mathrm{~kg} / \mathrm{m}^{2}$ were used to classify overweight and obesity, respectively. Adolescents were asked to self-report sex, smoking status, postal code to classify rural or urban residence, and ethnicity, categorized as white, Indigenous, or other.

\section{Statistical analyses}

Unadjusted comparisons of descriptive characteristics and cardiometabolic health outcomes were initially evaluated between adolescents with type 2 diabetes who participated in regular vigorous-intensity activity and those who did not, using Mann-Whitney U test, t-test, and $\chi^{2}$, where appropriate. Significant variables from the univariate analyses and/or variables a priori chosen for their association with cardiometabolic health outcomes were included in a multivariate regression with adjustment for sex, duration of diabetes, rural/urban status, BMI Z-score, and smoking. The secondary analyses were multivariate logistic regressions comparing the odds of hypertension and albuminuria between groups stratified by participation in regular vigorous-intensity activity, adjusting for sex, duration of diabetes, rural/urban status, BMI Z-score, and smoking. We also conducted a series of sensitivity analyses that included

Table 2 Demographic variables and cardiometabolic health outcomes among adolescents with type 2 diabetes stratified according to participation in vigorous-intensity physical activity

\begin{tabular}{llcc}
\hline Variable & No vigorous-intensity physical activity & Vigorous-intensity physical activity & P value \\
\hline $\mathrm{n}$ & 97 & 67 & 1.000 \\
\hline Female, $\mathrm{n}(\%)$ & $67(69.1)$ & $46(68.7)$ & 0.284 \\
\hline Rural, $\mathrm{n}(\%)$ & $79(81.4)$ & $49(73.1)$ & 1.00 \\
\hline Indigenous, $\mathrm{n}(\%)$ & $94(96.9)$ & $56(83.6)$ & 0.007 \\
\hline Smoking, $\mathrm{n}(\%)$ & $36(37.1)$ & $11(16.4)$ & 0.210 \\
\hline Mean age (years) & $15.32(2.79)$ & $14.81(2.08)$ & 0.324 \\
\hline Median duration of diabetes (years) & $2.50(1.53-4.63)$ & $2.53(1.07-3.96)$ & 0.191 \\
\hline Mean BMI Z-score & $2.31(1.07)$ & $2.53(1.09)$ & 0.016 \\
\hline Mean HbA1c (\%) & $10.0(2.42)$ & $9.01(2.74)$ & 0.05 \\
\hline Mean 24-hour SBP (mm Hg) & $126.0(9.3)$ & $122.9(10.1)$ & $<0.001$ \\
\hline Mean 24-hour DBP (mm Hg) & $73.2(5.5)$ & $70.0(6.1)$ & 0.003 \\
\hline Mean 24-hour MAP (mm Hg) & $90.3(6.1)$ & $87.3(6.1)$ & 0.189 \\
\hline Median daytime systolic load (\%) & $28.1(15.4-55.3)$ & $23.6(9.0-48.2)$ & 0.052 \\
\hline Median daytime diastolic load (\%) & $15.9(6.8-25.8)$ & $10.10(3.7-20.8)$ & 0.030 \\
\hline Mean night-time systolic load & $55.7(32.4)$ & $43.87(31.2)$ & 0.850 \\
\hline Mean LDL (mmol/L) & $2.38(0.77)$ & $2.36(0.56)$ & 0.841 \\
\hline Mean HDL (mmol/L) & $1.18(0.38)$ & $1.17(0.28)$ & 0.901 \\
\hline Median triglycerides (mmol/L) & $1.40(1.00-2.70)$ & $1.60(1.00-2.10)$ & $<0.001$ \\
\hline Physical activity (MET-hour/week) & $34.3(13.4-80.6)$ & $62.4(37.7-121.4)$ & \\
\hline Dare pres) & & \\
\hline
\end{tabular}

Data are presented as mean (SD) for normally distributed variables and median (IQR) for non-normally distributed variables.

BMI, body mass index; DBP, diastolic blood pressure; HbA1c, hemoglobin A1c; HDL, high-density lipoprotein; LDL, low-density lipoprotein;

MAP, mean arterial pressure; MET, metabolic equivalent; SBP, systolic blood pressure. 
multivariate linear regression testing for differences in cardiometabolic health outcomes between youth categorized as physically active or not, based on other definitions (meeting guidelines of $60 \mathrm{~min}$ of moderate-intensity to vigorous-intensity activity weekly or participation in any organized sport or not). All continuous variables were not normally distributed and the results are therefore presented as median with first and third quartiles. Participants with missing data were not included in the analyses and we did not use imputation to account for missing data. All data were analyzed using R V.3.4.1 and SPSS V.26. Between-group differences with an $\alpha<0.05$ were considered statistically different.

\section{RESULTS}

The 164 adolescents included in the analysis were $15 \pm 3$ years old, and $78 \%$ lived rurally and $68 \%$ were girls, with a mean BMI Z-score of $2.4 \pm 1.1$ and HbAlc of $9.6 \% \pm 2.6 \%$ (table 1), and $41 \%$ reported participating in vigorous-intensity physical activity organized activities. Adolescents who participated in regular vigorous-intensity physical activity were less likely to smoke than those who did not $(\mathrm{p}<0.05)$. Both groups were comparable in age, sex, and duration of diabetes, were likely to live rurally, and of Indigenous descent (table 2). Adolescents who reported participating in vigorous-intensity physical activity achieved nearly twice the dose of physical activity weekly than less active peers (62 vs 34 MET-hour/week, $\mathrm{p}=0.001$ ). No differences in mean BMI Z-score or lipids were observed between the groups. In unadjusted analyses, the odds of albuminuria (OR: $0.36,95 \%$ CI 0.17 to 0.74 ), HbA1c $(9.0 \%$ vs $10.0 \%)$, 24-hour mean systolic (122.9 mm $\mathrm{Hg}$ vs $126.0 \mathrm{~mm} \mathrm{Hg}$ ) and diastolic $(70.0 \mathrm{~mm} \mathrm{Hg}$ vs 73.2 $\mathrm{mm} \mathrm{Hg}$ ) blood pressure, and mean arterial pressure (87.3 $\mathrm{mm} \mathrm{Hg}$ vs $90.3 \mathrm{~mm} \mathrm{Hg}$ ) were all lower in adolescents who reported participating in vigorous-intensity physical activity compared with peers who did not.

In fully adjusted linear regression analyses, differences in HbAlc were no longer significantly different between groups $(9.1 \%$ vs $9.9 \%, \mathrm{p}=0.052)$; however, differences in a number of measures of blood pressure remained significantly different. Specifically, mean diastolic and mean arterial pressures during both wake and sleep recordings were significantly lower in adolescents who reported participation in regular vigorous-intensity physical activity (table 3 ). Mean systolic blood pressure during sleep was also significantly lower in adolescents who participated in vigorous-intensity physical activity, compared with those who did not (113.6 $\mathrm{mm} \mathrm{Hg}$ vs $118 \mathrm{~mm} \mathrm{Hg}, \mathrm{p}=0.023$ ). Despite differences in wake and sleep blood pressure, the odds of hypertension were not different between the groups in fully adjusted logistic regression analyses (table 3). The odds of albuminuria were $60 \%$ lower (adjusted OR: 0.40, 95\% CI 0.19 to 0.84 ) and the odds of $\mathrm{HbAl} 1 \mathrm{c}>9 \%$ were $50 \%$ lower (adjusted OR: $0.50,95 \%$ CI 0.26 to 0.98 ) among adolescents who engaged in regular vigorous-intensity physical activity, compared with those who did not (table 4).

In sensitivity analyses, no differences in any of the outcomes studied were observed between adolescents with type 2 diabetes stratified by meeting recommendations for weekly physical activity (>40 MET-hour/week) or when stratified by participation in any organized physical activity (online supplemental table 1 and 2).

\section{DISCUSSION}

To the best of our knowledge, this is the first study that tested for an association between vigorous-intensity physical activity and cardiometabolic health outcomes in adolescents with type 2 diabetes. The main findings are

Table 3 Differences in ambulatory blood pressure readings between adolescents living with type 2 diabetes stratified according to participation in vigorous-intensity physical activity

\begin{tabular}{lccc}
\hline Variable & No vigorous-intensity physical activity & Vigorous-intensity physical activity & P value \\
\hline $\mathrm{n}$ & 87 & 66 & \\
\hline Wake SBP $(\mathrm{mm} \mathrm{Hg})$ & $128.1(126.1$ to 130.0$)$ & $125.7(123.5$ to 128.0$)$ & 0.127 \\
\hline Wake DBP $(\mathrm{mm} \mathrm{Hg})$ & $75.2(73.9$ to 76.5$)$ & $72.6(71.1$ to 74.1$)$ & $\mathbf{0 . 0 1 0}$ \\
\hline Wake MAP $(\mathrm{mm} \mathrm{Hg})$ & $92.1(90.7$ to 93.4$)$ & $89.5(87.9$ to 91.0$)$ & $\mathbf{0 . 0 1 4}$ \\
\hline Wake SBP load $(\%)$ & $34.4(28.9$ to 39.9$)$ & $31.6(25.2$ to 37.9$)$ & 0.515 \\
\hline Wake DBP load $(\%)$ & $17.9(14.5$ to 21.3$)$ & $15.8(11.9$ to 19.7$)$ & 0.437 \\
Sleep SBP $(\mathrm{mm} \mathrm{Hg})$ & $118.0(115.5$ to 120.5$)$ & $113.6(110.7$ to 116.4$)$ & $\mathbf{0 . 0 2 3}$ \\
\hline Sleep DBP $(\mathrm{mm} \mathrm{Hg})$ & $65.3(63.7$ to 67.0$)$ & $61.1(59.2$ to 63.0$)$ & $\mathbf{0 . 0 0 1}$ \\
\hline Sleep MAP $(\mathrm{mm} \mathrm{Hg})$ & $83.0(81.3$ to 84.7$)$ & $79.3(77.3$ to 81.3$)$ & $\mathbf{0 . 0 0 6}$ \\
\hline Sleep SBP load $(\%)$ & $54.8(47.7$ to 61.9$)$ & $44.9(36.7$ to 53.1$)$ & 0.077 \\
Sleep DBP load $(\%)$ & $38.2(32.4$ to 22.0$)$ & $26.3(19.6$ to 33.0$)$ & $\mathbf{0 . 0 1 0}$ \\
\hline
\end{tabular}

Adjusted for sex, duration of diabetes, and rural/urban status.

Data are presented as adjusted mean and 95\% Cl (in parentheses).

$\mathrm{p}$ values represent results from groupwise comparison between participants reporting no vigorous intensity physical activity and those reporting vigorous-intensity physical activity.

DBP, diastolic blood pressure; MAP, mean arterial pressure; SBP, systolic blood pressure. 
Table 4 Multivariate logistic regression analyses describing the odds of various cardiometabolic outcomes between adolescents with type 2 diabetes stratified by activity levels

\begin{tabular}{|c|c|c|c|c|}
\hline Variable & $\begin{array}{l}\text { No vigorous-intensity } \\
\text { physical activity }\end{array}$ & $\begin{array}{l}\text { Vigorous-intensity } \\
\text { physical activity }\end{array}$ & OR $(95 \% \mathrm{Cl})$ & $\begin{array}{l}\text { Adjusted OR (95\% } \\
\mathrm{Cl})\end{array}$ \\
\hline Day hypertension, n (\%) & $23(26.4)$ & $12(18.5)$ & $0.63(0.29$ to 1.38$)$ & 0.59 (0.26 to 1.34$)$ \\
\hline Night hypertension, n (\%) & $47(58.8)$ & $25(41.0)$ & $0.49(0.25$ to 0.97$)$ & $0.54(0.27$ to 1.07$)$ \\
\hline Night dipping hypertension, n (\%) & $58(72.5)$ & $37(60.7)$ & $0.59(0.29$ to 1.19$)$ & $0.68(0.32$ to 1.41$)$ \\
\hline Any hypertension, n (\%) & $51(60.7)$ & $29(47.5)$ & $0.59(0.30$ to 1.14$)$ & $0.64(0.32$ to 1.27$)$ \\
\hline ACR >2 mg/mmol, $\mathrm{n}(\%)$ & $39(40.2)$ & $13(19.4)$ & $0.36(0.17$ to 0.74$)$ & $0.40(0.19$ to 0.84$)$ \\
\hline $\mathrm{HbA} 1 \mathrm{c}>9 \%, \mathrm{n}(\%)$ & $60(62.5)$ & $29(43.3)$ & $0.46(0.24$ to 0.87$)$ & $0.50(0.26$ to 0.98$)$ \\
\hline
\end{tabular}

OR: unadjusted odds with $95 \% \mathrm{Cl}$; adjusted OR: same odds, but adjusted for differences in sex, smoking, duration of diabetes, rural/urban status, and body mass index Z-score.

$\mathrm{ACR}$, albumin to creatinine ratio; HbA1c, hemoglobin A1c.

that adolescents living with type 2 diabetes who participate in regular vigorous-intensity physical activity are characterized by lower blood pressure, modestly better glycemic control, and reduced odds of albuminuria compared with peers who participate in less vigorous physical activity. Additionally, these differences in cardiometabolic health outcomes were not observed when adolescents were stratified according to meeting current guidelines for weekly physical activity or simply by participation in organized sport. These data provide preliminary evidence for a potential role of vigorous-intensity physical activity in the management of cardiometabolic health outcomes among adolescents with type 2 diabetes.

Participation in regular physical activity is associated with an array of cardiometabolic health benefits for adolescents without diabetes. ${ }^{29}$ Previous studies suggest that participation in regular physical activity is associated with better glycemic control, lipoprotein profiles, and lower arterial stiffness among youth ${ }^{16} 17$ and adults living with type 2 diabetes. ${ }^{6}$ Among youth without diabetes, the cardiometabolic health benefits of physical activity appear to be greater with vigorousintensity physical activity, relative to light-intensity or moderate-intensity physical activity. ${ }^{14}$ 15 The data presented here extend these findings by demonstrating that the favorable association between vigorous-intensity physical activity and cardiometabolic health outcomes is also evident in adolescents with type 2 diabetes. In a community-based cohort of adolescents without chronic disease, we documented that accumulating daily bouts of vigorous-intensity physical activity was associated with a $44 \%$ lower odds of high normal blood pressure, ${ }^{15}$ which was sustained for 2 years. ${ }^{14}$ Using a self-reported instrument of physical activity, we expand this observation to a cohort of adolescents with type 2 diabetes, who displayed 3-5 $\mathrm{mm} \mathrm{Hg}$ lower mean diastolic blood pressure and mean arterial pressure coupled with 50\% lower odds of albuminuria, compared with peers who do not engage in regular vigorous-intensity physical activity. Similar to previous studies of vigorous-intensity physical activity and cardiometabolic health outcomes among youth without diabetes, ${ }^{14}{ }^{15}$ these associations were not evident with lower-intensity physical activity. These data reinforce the importance of vigorous-intensity physical activity in the management of cardiometabolic risk factors among adolescents with type 2 diabetes.

Renal injury is a frequent complication in adolescents with type 2 diabetes ${ }^{30} 31$ and predicts long-term chronic kidney disease. ${ }^{32}$ Significant weight loss, secondary to bariatric surgery, is associated with resolution of proteinuria in adolescents with type 2 diabetes. ${ }^{33}$ Little evidence exists on the role of modifiable lifestyle behaviors in the management of albuminuria in adolescents with type 2 diabetes. The current study provides preliminary observational evidence that regular vigorous-intensity physical activity, but not moderate-intensity or light-intensity physical activity, may provide renal protection in adolescents with type 2 diabetes. The lower odds of albuminuria are likely a function of lower blood pressure and modestly better glycemic control among active adolescents, rather than a direct effect of physical activity. Physical activity is associated with acute increases in albumin excretion; however, regular daily activity confers health benefits in individuals with type 1 diabetes, including those with renal injury. ${ }^{34}$ Prospective cohort studies and experimental trials with prolonged follow-up are needed to confirm these cross-sectional observations.

Health behavior change for adolescents with type 2 diabetes is a significant clinical challenge. ${ }^{35}$ Adolescents with type 2 diabetes suffer from significant psychosocial barriers that influence their readiness for behavior change. ${ }^{36-38}$ In the current study, adolescents with type 2 diabetes who engaged in vigorous-intensity physical activity did so largely through school-based activities/ sports. Stratifying adolescents based on vigorous-intensity organized sport may have introduced selection bias ${ }^{39}$ and associated confounding, ${ }^{40}$ as readiness for engaging in these activities likely reflects more comprehensive adoption of healthy behaviors. From a clinical standpoint, however, encouraging and supporting adolescents with type 2 diabetes to engage in organized activities at school or in their communities could be a starting point to increase a sense of mastery and belonging ${ }^{37}$ and readiness for adopting health behaviors. 
The strengths of this study include the extensive phenotyping of cardiometabolic risk factors within a relatively large cohort of youth with type 2 diabetes. The study is however limited due to its cross-sectional design, precluding any causal inference regarding the observations described here. Furthermore, the data for physical activity were self-reported and therefore may be biased toward higher levels of daily activity. Therefore, these observations are at risk of a type 1 error and recall bias. Finally, as the majority of the adolescents included in the study were Indigenous and recruited from a single clinical center in Manitoba, Canada, the generalizability of these findings needs to be determined. Larger prospective cohort studies, using objective measurements of physical activity, within multiethnic samples, followed for years, are needed to confirm and expand on these preliminary observations.

\section{CONCLUSION}

Adolescents with type 2 diabetes who engage in regular vigorous-intensity physical activity display better cardiometabolic health profiles than peers who do not engage in similar physical activity. These associations are not evident with lower-intensity physical activity or simply engaging in organized sport. Randomized clinical trials or longitudinal, well-powered cohort studies of physical activity and cardiometabolic health in adolescents with type 2 diabetes are needed to confirm these observations.

Acknowledgements We are indebted to the families involved in the iCARE cohort for dedicating their time and energy to this study. We would also like to acknowledge the iCARE Patient Advisory Group (PAG) as partners in the iCARE cohort study.

Contributors JLS and JMM conceptualized the study, collected the data, helped with analyses, drafted the initial manuscript, and edited and finalized the manuscript. ABD, BAW, and EACS participated in the recruitment of participants, data collection, edited the manuscript for important intellectual content, and approved the final version of the manuscript. ABD and BAW are co-principal investigators for the iCARE cohort and secured funding for data collection and oversaw data collection and management of the study. MG, MC, and JLS participated in data collection and data management, helped write the methods section of this manuscript, and edited the final version of the manuscript. JMM and JLS performed the statistical analyses. JMM is the guarantor and takes full responsibility for the content of the article.

Funding This work was funded by grants awarded to our research team from the Heart and Stroke Foundation of Canada, Diabetes Canada, and CIHR.

Competing interests JLS, JMM, ABD, EACS, MG, and MC have no financial conflicts to disclose. BAW has received funding from CIHR, Diabetes Canada, the Children's Hospital Research Institute of Manitoba (CHRIM), and the Lawson Foundation for unrelated investigator-led studies. She is currently the site PI for a Boehringer Ingelheim study unrelated to the current study content. She has not received monetary support personally from pharmaceutical companies. EACS has received funding from Diabetes Canada, CHRIM, and the Public Health Agency of Canada for unrelated investigator-led studies. ABD currently has research funding from CIHR, Research Manitoba, and CHRIM. JMM, EACS, BAW, and ABD were also supported by a Research Manitoba grant to the DEVOTION research cluster. None of the cited agencies was involved in the design, conduct, or approval of this manuscript. JMM holds grants from the CIHR, Diabetes Canada, Children's Hospital Foundation of Manitoba, the Heart and Stroke Foundation of Canada (HSFC), the Cosmopolitan Foundation, and the Lawson Foundation.

Patient consent for publication Not required.
Ethics approval The University of Manitoba Research Ethics Board approved the study protocol.

Provenance and peer review Not commissioned; externally peer reviewed.

Data availability statement Data are available upon reasonable request. Summary data are available upon request to the authors and are contingent upon approval from patient advisory and Indigenous data advisory committee for the iCARE cohort and DREAM theme, respectively. Requests can be made to dream@ umanitoba.ca with reference to iCARE cohort study.

Supplemental material This content has been supplied by the author(s). It has not been vetted by BMJ Publishing Group Limited (BMJ) and may not have been peer-reviewed. Any opinions or recommendations discussed are solely those of the author(s) and are not endorsed by BMJ. BMJ disclaims all liability and responsibility arising from any reliance placed on the content. Where the content includes any translated material, BMJ does not warrant the accuracy and reliability of the translations (including but not limited to local regulations, clinical guidelines, terminology, drug names and drug dosages), and is not responsible for any error and/or omissions arising from translation and adaptation or otherwise.

Open access This is an open access article distributed in accordance with the Creative Commons Attribution Non Commercial (CC BY-NC 4.0) license, which permits others to distribute, remix, adapt, build upon this work non-commercially, and license their derivative works on different terms, provided the original work is properly cited, appropriate credit is given, any changes made indicated, and the use is non-commercial. See: http://creativecommons.org/licenses/by-nc/4.0/.

ORCID iDs

Brandy Alexandra Wicklow http://orcid.org/0000-0002-6080-0494

Jonathan M McGavock http://orcid.org/0000-0002-3741-5248

\section{REFERENCES}

1 TODAY Study Group, Zeitler P, Hirst K, et al. A clinical trial to maintain glycemic control in youth with type 2 diabetes. $N$ Engl $J$ Med 2012;366:2247-56.

2 Inge TH, Miyano G, Bean J, et al. Reversal of type 2 diabetes mellitus and improvements in cardiovascular risk factors after surgical weight loss in adolescents. Pediatrics 2009;123:214-22.

3 Huynh E, Rand D, McNeill C, et al. Beating diabetes together: a mixed-methods analysis of a feasibility study of intensive lifestyle intervention for youth with type 2 diabetes. Can J Diabetes 2015;39:484-90.

4 Wittmeier KD, Wicklow BA, Sellers EA, et al. Success with lifestyle monotherapy in youth with new-onset type 2 diabetes. Paediatr Child Health 2012;17:129-32.

5 Marcus MD, Wilfley DE, El Ghormli L, et al. Weight change in the management of youth-onset type 2 diabetes: the today clinical trial experience. Pediatr Obes 2017;12:337-45.

6 Umpierre D, Ribeiro PAB, Kramer CK, et al. Physical activity advice only or structured exercise training and association with $\mathrm{HbA} 1 \mathrm{c}$ levels in type 2 diabetes: a systematic review and meta-analysis. JAMA 2011;305:1790-9.

7 Adolfsson P, Riddell MC, Taplin CE, et al. ISPAD clinical practice consensus guidelines 2018: exercise in children and adolescents with diabetes. Pediatr Diabetes 2018;19 Suppl 27:205-26.

8 Diabetes Canada Clinical Practice Guidelines Expert Committee, Panagiotopoulos C, Hadjiyannakis S, et al. Type 2 diabetes in children and adolescents. Can J Diabetes 2018;42 Suppl 1:S247-54.

9 American Diabetes Association. 13. Children and Adolescents: Standards of Medical Care in Diabetes-2020. Diabetes Care 2020;43:S163-82.

10 Wittmeier KDM, Wicklow BA, Maclntosh AC, et al. Hepatic steatosis and low cardiorespiratory fitness in youth with type 2 diabetes. Obesity 2012;20:1034-40.

11 Shaibi GQ, Michaliszyn SB, Fritschi C, et al. Type 2 diabetes in youth: a phenotype of poor cardiorespiratory fitness and low physical activity. Int J Pediatr Obes 2009;4:332-7.

12 Lobelo F, Liese AD, Liu J, et al. Physical activity and electronic media use in the search for diabetes in youth case-control study. Pediatrics 2010;125:e1364-71.

13 Renninger M, Hansen BH, Steene-Johannessen J, et al. Associations between accelerometry measured physical activity and sedentary time and the metabolic syndrome: a meta-analysis of more than 6000 children and adolescents. Pediatr Obes 2020;15:e12578. 
14 Carson V, Rinaldi RL, Torrance B, et al. Vigorous physical activity and longitudinal associations with cardiometabolic risk factors in youth. Int J Obes 2014;38:16-21.

15 Hay J, Maximova K, Durksen A, et al. Physical activity intensity and cardiometabolic risk in youth. Arch Pediatr Adolesc Med 2012;166:1022-9.

16 Herbst A, Kapellen T, Schober E, et al. Impact of regular physical activity on blood glucose control and cardiovascular risk factors in adolescents with type 2 diabetes mellitus--a multicenter study of 578 patients from 225 centres. Pediatr Diabetes 2015;16:204-10.

17 Wittekind SG, Edwards NM, Khoury PR, et al. Association of habitual physical activity with cardiovascular risk factors and target organ damage in adolescents and young adults. Journal of Physical Activity and Health 2018;15:176-82.

18 Dart AB, Wicklow BA, Sellers EA, et al. The improving renal complications in adolescents with type 2 diabetes through the research (iCARE) cohort study: rationale and protocol. Can $J$ Diabetes 2014;38:349-55.

19 Vandenbroucke JPet al. Strengthening the reporting of observational studies in epidemiology (STROBE): explanation and elaboration. Ann Intern Med 2007;147:W-194.

20 Sellers E, Eisenbarth G, Young TK, et al. Diabetes-Associated autoantibodies in Aboriginal children. Lancet 2000;355:1156.

21 Mashford-Pringle A, Pavagadhi K. Using OCAP and IQ as frameworks to address a history of trauma in Indigenous health research. AMA J Ethics 2020;22:E868-73.

22 Booth ML, Okely AD, Chey TN, et al. The reliability and validity of the adolescent physical activity recall questionnaire. Med Sci Sports Exerc 2002;34:1986-95.

23 Ainsworth BE, Haskell WL, Whitt MC, et al. Compendium of physical activities: an update of activity codes and Met intensities. Med Sci Sports Exerc 2000;32:S498-516.

24 Puyau MR, Adolph AL, Vohra FA, et al. Prediction of activity energy expenditure using accelerometers in children. Med Sci Sports Exerc 2004;36:1625-31.

25 Soergel M, Kirschstein M, Busch C, et al. Oscillometric twentyfour-hour ambulatory blood pressure values in healthy children and adolescents: a multicenter trial including 1141 subjects. $J$ Pediatr 1997;130:178-84.

26 Dart AB, Wicklow B, Blydt-Hansen TD, et al. A holistic approach to risk for early kidney injury in Indigenous youth with type 2 diabetes: a proof of concept paper from the iCARE cohort. Can J Kidney Health Dis 2019;6:2054358119838836:205435811983883.

27 (WHO) WHO. BMI-for-age (5-19 years) 2020.

28 Rodd C, Sharma AK. Recent trends in the prevalence of overweight and obesity among Canadian children. CMAJ 2016;188:E313-20.

29 Ekelund U, Luan Jian'an, Sherar LB, et al. Moderate to vigorous physical activity and sedentary time and cardiometabolic risk factors in children and adolescents. JAMA 2012;307:704-12.

30 Dart AB, Wicklow B, Scholey J, et al. An evaluation of reninangiotensin system markers in youth with type 2 diabetes and associations with renal outcomes. Pediatr Diabetes 2020;21:1102-9.

31 TODAY Study Group. Rapid rise in hypertension and nephropathy in youth with type 2 diabetes: the today clinical trial. Diabetes Care 2013;36:1735-41.

32 Dart AB, Sellers EA, Martens PJ, et al. High burden of kidney disease in youth-onset type 2 diabetes. Diabetes Care 2012;35:1265-71.

33 Bjornstad P, Hughan K, Kelsey MM, et al. Effect of surgical versus medical therapy on diabetic kidney disease over 5 years in severely obese adolescents with type 2 diabetes. Diabetes Care 2020;43:187-95.

34 Tikkanen-Dolenc H, Wadén J, Forsblom C, et al. Physical activity reduces risk of premature mortality in patients with type 1 diabetes with and without kidney disease. Diabetes Care 2017;40:1727-32.

35 Kaar JL, Schmiege SJ, Drews K, et al. Evaluation of the longitudinal change in health behavior profiles across treatment groups in the today clinical trial. Pediatr Diabetes 2020;21:224-32.

36 Walders-Abramson N, Venditti EM, levers-Landis CE, et al. Relationships among stressful life events and physiological markers, treatment adherence, and psychosocial functioning among youth with type 2 diabetes. J Pediatr 2014;165:e501:504-8.

37 McGavock J, Durksen A, Wicklow B, et al. Determinants of readiness for adopting healthy lifestyle behaviors among Indigenous adolescents with type 2 diabetes in Manitoba, Canada: a crosssectional study. Obesity 2018;26:910-5.

38 McGavock J, Wicklow B, Dart AB. Type 2 diabetes in youth is a disease of poverty. Lancet 2017;390:1829.

39 Hernán MA, Hernández-Díaz S, Robins JM. A structural approach to selection bias. Epidemiology 2004;15:615-25.

40 Wade KH, Richmond RC, Davey Smith G. Physical activity and longevity: how to move closer to causal inference. Br J Sports Med 2018;52:890-1. 